\title{
Radiotherapy-associated angiosarcoma in the breast reconstructed by autologous free-flap and treated with electrochemotherapy
}

\author{
Romi Cencelj-Arnez ${ }^{1,2}$, Jerica Novak ${ }^{1,2}$, Andreja Klevisar Ivancic ${ }^{3}$, Masa Bosnjak \\ Maja Cemazar ${ }^{4,5}$, Marko Snoj ${ }^{1,2}$ \\ 1 Department of Surgical Oncology, Institute of Oncology Ljubljana, Ljubljana, Slovenia \\ 2 Faculty of Medicine, University of Ljubljana, Ljubljana, Slovenia \\ ${ }^{3}$ Department of Pathology, Institute of Oncology Ljubljana, Ljubljana, Slovenia \\ ${ }^{4}$ Department of Experimental Oncology, Institute of Oncology Ljubljana, Ljubljana, Slovenia \\ ${ }^{5}$ University of Primorska, Faculty of Health Sciences, Izola, Slovenia
}

Radiol Oncol 2021; 55(1): 77-81.

Received 20 October 2020

Accepted 27 November 2020

Correspondence to: Prof. Marko Snoj, M.D., Ph.D. Institute of Oncology Ljubljana, Department of Surgical Oncology, Zaloška 2, SI-1000 Ljubljana, Slovenia. E-mail: msnoj@onko-i.si

Disclosure: No potential conflicts of interest were disclosed.

\begin{abstract}
Background. Radiotherapy-associated angiosarcoma (RAA) of the breast is a rare complication of radiotherapy, which is often difficult to identify and has poor prognosis. It usually presents as violaceous skin, erythema or rapidly growing palpable firm mass that can be confused with other benign skin lesions.

Patients and methods. After reviewing the literature, we found only four cases with RAA after mastectomy and autologous breast reconstruction. The presented case is the first that was treated by electrochemotherapy. The patient presented with secondary angiosarcoma of the breast five years after mastectomy, immediate breast reconstruction with deep inferior epigastric artery perforator free flap and adjuvant radiotherapy.

Results. Electrochemotherapy was feasible, safe and effective in treatment of radiation induced sarcoma. Most of the treated lesions in several consecutive electrochemotherapy sessions responded with complete response, but multiple recurrences occurred in non-treated areas.

Conclusions. Patients with breast cancer after skin-sparing mastectomy and immediate breast reconstruction, who receive radiotherapy, need regular long-term follow up and low threshold for biopsy of any suspicious lesions is mandatory. Electrochemotherapy proved as one of feasible modalities of treatment for RAA.
\end{abstract}

Key words: radiation-associated angiosarcoma; breast reconstruction; breast cancer; electrochemotherapy; radiotherapy

\section{Introduction}

Angiosarcoma of the breast is a rare malignancy, which can occur as primary without a known precursor or as secondary from associated radiotherapy or chronic lymphedema. ${ }^{1}$ Although radiotherapy-associated angiosarcoma (RAA) is a rare complication of radiotherapy, it is the most frequent classifiable sarcoma arising in women with breast cancer treated with radiotherapy in the field of irradiated soft tissue. ${ }^{2}$ The diagnosis is usually late because initial signs of RAA may be subtle and difficult to identify. Cahn et al. suggested the following criteria for the diagnosis of RAA: the sarcoma should arise in the area previously subjected to irradiation, a latent period (in years) must exist between the time of irradiation and development of the sarcoma, and the sarcoma must be confirmed histologically. ${ }^{3}$

Radiotherapy continues to be a mainstay modality in the treatment of breast cancer patients after breast conserving surgery and has a significant role 
in preventing local recurrence of breast tumors. ${ }^{1}$ After reviewing the literature, we found most of the cases with RAA after breast conserving surgery and only four cases with RAA after mastectomy and autologous breast reconstruction. ${ }^{4-6}$ We report a case of RAA after bilateral skin-sparing mastectomy and immediate free flap breast reconstruction to highlight awareness of the disease in an autologous breast reconstruction and the importance of early detection. Furthermore, we want to show that electrochemotherapy, might be applied even in such a rare condition in the palliative intent.?

\section{Case report and literature review}

A 63-year-old Caucasian female presented with central violaceous or hyperpigmented macule surrounded by an erythematous ring in the lower inner quadrant of her right breast measuring $3 \times 5 \mathrm{~cm}$ (Figure 1) in June 2016. She had a history of synchronous bilateral hormone positive HER-2 positive breast carcinoma with positive lymph nodes in the right axilla six years ago. After six cycles of the neoadjuvant 5-Fluoro uracil, epirubicin and cyclophosphamide (FEC-100) chemotherapy bilateral skin-sparing mastectomy, axillary lymph node dissection on the right and sentinel node biopsy on the left side followed, and immediate bilateral breast reconstruction with deep inferior epigastric perforator (DIEP) flap was performed in April 2010.

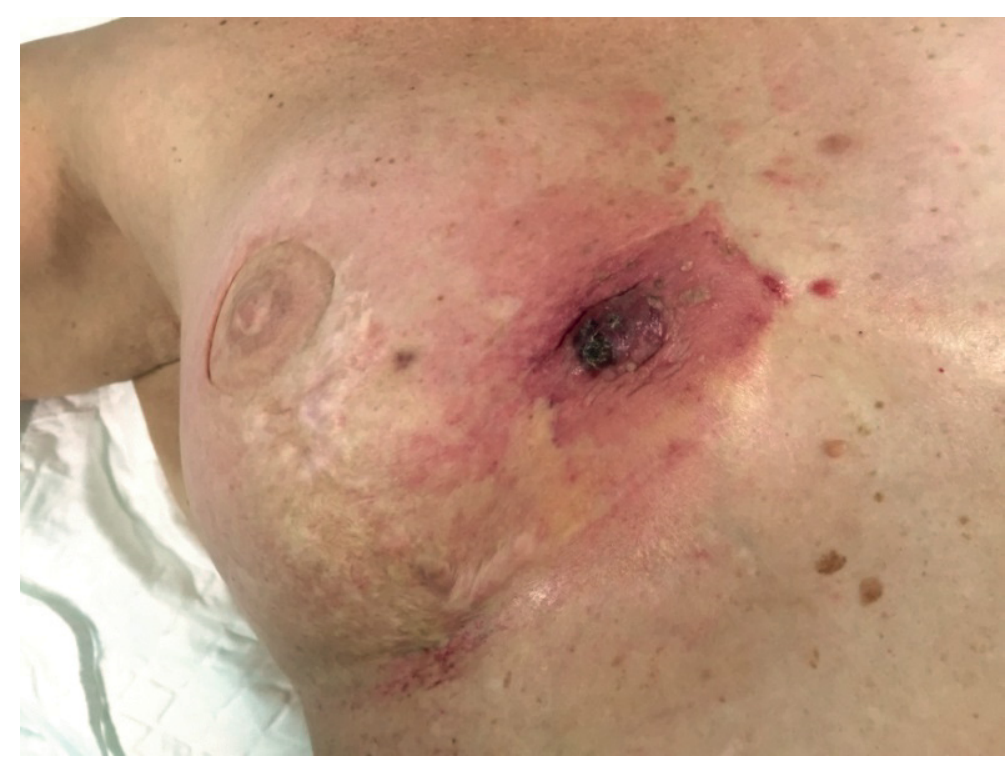

FIGURE 1. Clinical presentation of patient with angiosarcoma of right reconstructed breast.
Histology showed pathologic complete response of the tumor in the breast, with less than $10 \%$ of tumor cells and complete response in the right axilla. Postoperative irradiation of the right mammary region was done with 25 Gy in 2 Gy fractions followed by adjuvant hormonal treatment with letrozole for five years and later extended adjuvant hormonal therapy with tamoxifen.

She had regular follow up and was doing well until the lesion in her right breast was presented in October 2015. First, it was treated as fungal infection with topical ointments and later with corticosteroid ointments. Eight months after the first presentation, fine needle aspiration biopsy was done and suggested a melanocytic lesion, possible melanoma. In June 2016, excision of the lesion was performed and pathological examination revealed RAA, high grade, multinodular, involving dermis and free flap, infiltrating lateral resection margins (Figure 2). Amplification of the MYC oncogene was confirmed using fluorescence in situ hybridization (FISH) method (Figure 3). Patient underwent six cycles of chemotherapy with paclitaxel followed by wide resection of the affected area in January 2017. Pathological examination revealed multifocal residual angiosarcoma in area of $10 \mathrm{~cm}$ in diameter, involving skin and subcutaneous tissue with at least $1 \mathrm{~cm}$ clear resection margins. Four months after the last operation, in May 2017, she developed second local recurrence with multiple small skin lesion around the scar of the right breast. She received second-line chemotherapy with liposomal doxorubicin with stagnation of the lesions.

In October 2017 resection of the largest skin lesion in combination with electrochemotherapy (ECT) of the smaller 6 skin lesions (indicated in Figure 4 ), with diameter $0.8-1.2 \mathrm{~cm}$ and thickness

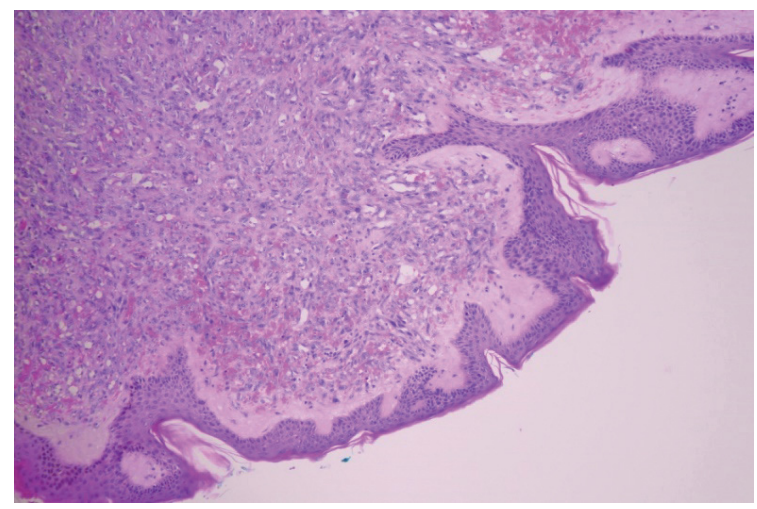

FIGURE 2. Pathologic hematoxylin and eosin (HE) specimen showing radiotherapy-associated angiosarcoma (RAA) involving dermis. 


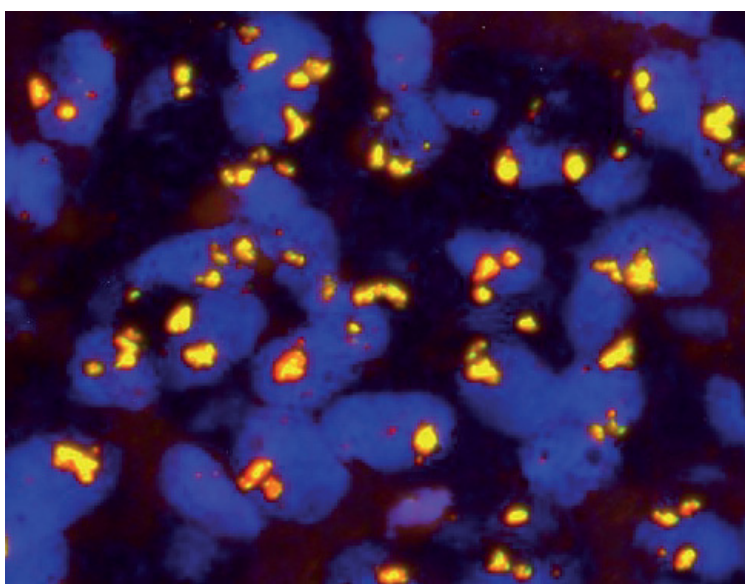

FIGURE 3. Amplification of the MYC oncogene was confirmed using FISH method on tumor specimen.

$0.2-0.4 \mathrm{~cm}$, was performed (Figure 4). ECT was performed 8 minutes after i.v. bleomycin infusion (30,000 IU) lasting 2 minutes using hexagonal electrodes and Cliniporator electric pulse generator (IGEA, s.r.l., Carpi, Italy) according to the SOP. 8,9 Trains of 8 electric pulses $(1000 \mathrm{~V} / \mathrm{cm})$, each pulse $100 \mu \mathrm{s}$ long, were delivered to each pair of electrodes consecutively. Altogether 7 electric pulse applications were performed. The patient had complete regression of the area treated 1 month after ECT. In December 2017, two months after the ECT treatment there was a third recurrence outside of the treated area. While other lesions were in complete regression also 2 months after ECT one of the treated lesions recurred. Fine needle biopsy of new and recurred lesion confirmed angiosarcoma metastases. Therefore, additional ECT was performed in January 2018 on 7 lesions, resulting in partial response of 1 lesion, stable disease of 1 lesion and complete response of other 5 lesions, 2 months after the treatment. Treatment was performed in the same way as in the first ECT session. Nevertheless, several new lesions occurred. The lesions were approximately $0.4 \mathrm{~cm}$ in diameter and scattered throughout the chest. Although the edema was present at several parts of the chest skin, ECT was performed once again in order to palliate the symptoms in March 2018. Plate electrodes were used to treat 40 small nodules. At next follow up, 1 month after the third ECT session all treated nodules regressed (most of them were in complete response and some of them in partial response). As already observed, new recurrences occurred in an interval less than one month after the last ECT and she received third line of chemotherapy with gemcitabine in May 2018. She received also four lines

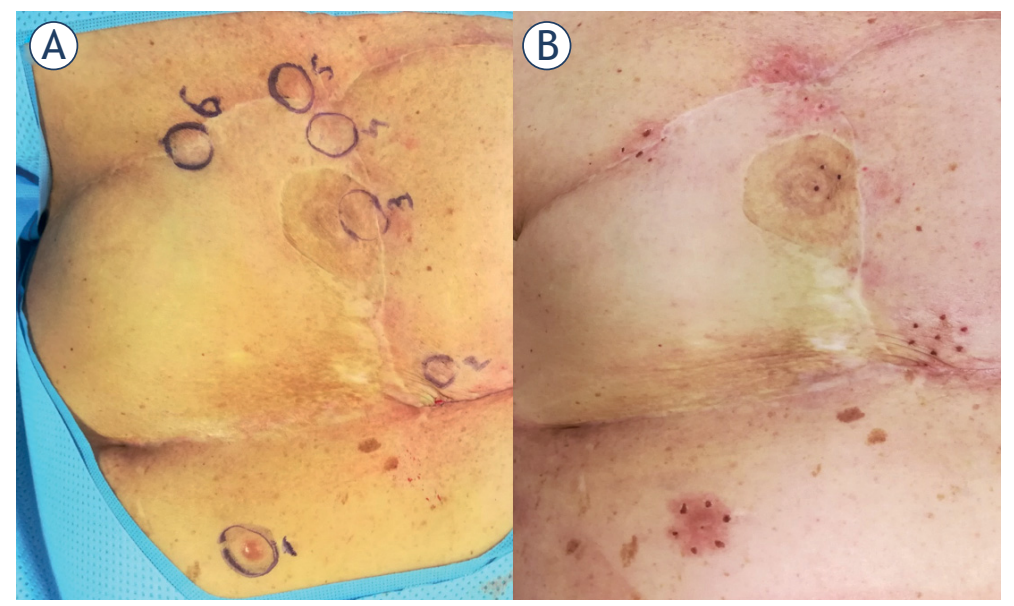

FIGURE 4. Treatment with electrochemotherapy. (A) Just before the treatment nodules were marked for evaluation; (B) 1 month after the treatment all nodules were in complete response.

of target therapy, but despite our best effort she died as a consequence of local tumor progression in May 2019, nearly 36 months after RAA presentation.

While the development of secondary angiosarcoma due to radiation is not uncommon in patients who underwent lumpectomy, the development of RAA in reconstructed breast is extremely rare with only four other cases reported in the literature.-6 In all four cases, RAA developed after more than 5 years after radiotherapy. In three cases modified radical mastectomy was done. In all cases the type of reconstruction was autologous - free transverse rectus abdominis muscle (TRAM), free deep inferior epigastric artery perforator (DIEP) flap as in our case and one case of a pedicled flap. In all reported cases, surgery was the most important, and in all but one, the only treatment modality. Table 1 represents the summary of case reports with RAA in the reconstructed breast.

\section{Discussion}

Radiotherapy plays an important role for many patients diagnosed with breast cancer to reduce local recurrence after breast-conserving surgery, rarely after mastectomy in specific cases. However, it is associated with increased risk of developing a secondary cancer, including tumors of the skin, such as basal cell carcinoma, squamous cell carcinoma, melanoma and angiosarcoma. ${ }^{10}$

Radiation-associated angiosarcoma of the breast often appears as violaceous skin, erythema, palpa- 
TABLE 1. Case reports with radiotherapy-associated angiosarcoma (RAA) after autologous breast reconstruction

\begin{tabular}{llllll}
\hline First author & Year published & Case \# & Years after RT & Type of cancer surgery & Type of breast reconstruction \\
\hline Hanasono et al. ${ }^{4}$ & 2005 & 1 & 6 & mastectomy & DIEP flap \\
Aljarrah et al. ${ }^{5}$ & 2014 & 2 & 6 & mastectomy & TRAM flap \\
Yip et al. ${ }^{6}$ & 2019 & 3 & 5 & mastectomy and axillary lymph node dissection & LD flap \\
Yip et al. ${ }^{6}$ & 2019 & 4 & 10 & mastectomy and axillary lymph node dissection & DIEP flap \\
\hline
\end{tabular}

DIEP = deep inferior epigastric artery perforator; $M R M=$ modified radical mastectomy; RT = radiotherapy; $T R A M=$ transverse rectus abdominis muscle; $L D=$ latissimus dorsi muscle

ble firm mass, skin thickening or skin dimpling, papules and edema that can be confused with other benign skin lesions, such as telangiectasia or changes associated with trauma. ${ }^{1,11}$ Atypical postradiation vascular proliferation (APRVP) is one of vascular radiation-associated lesion that shares similar clinical and histologic features to RAA. The vast majority of APRVP has benign clinical course, although coexistence of APRVP and RAA has been described in some reports and raises the possibility that APRVP represents a precursor of RAA although this theory remains controversial. ${ }^{12}$ Immunohistochemical staining and FISH method for MYC are helpful in distinguishing benign and atypical vascular lesions from RAA since amplification of MYC is present in more than $90 \%$ of secondary angiosarcomas and is absent in atypical vascular lesions. ${ }^{1}$ Subtle clinical signs and rare occurrence of the disease often delay the diagnosis as in our case, so the diagnosis of atypical vascular lesions in violaceous or erythematous skin in irradiated breast should prompt further investigation to rule out RAA. Excision biopsy and pathological examination is necessary for definitive diagnosis .

Angiosarcoma of the breast can develop as primary or secondary malignancy. The distinction between primary breast angiosarcoma and secondary RAA is that the former arises within the mammary parenchyma, whereas the latter is principally a dermal/subcutaneous lesion that may or may not invade the underlying parenchyma and is usually multifocal. ${ }^{11,13}$ In our case, the tumor invaded the dermis and also the parenchyma of the free flap. Partial fat necrosis, which usually presents as firm mass, with skin unchanged, is a known complication after breast reconstruction with DIEP flap and can misguide the diagnosis of RAA. ${ }^{14}$

Most of the RAA present as localized and multifocal disease. The median time from the administration of RT for primary breast cancer to the diagnosis of angiosarcoma ranges from four to 11 years, on average 7 years..$^{15}$ The presented case and all four reported cases developed RAA five years or more after radiotherapy. Therefore, careful patient evaluation for early disease detection in long-term follow up of patients that received radiotherapy even in cases of autologous breast reconstruction for breast cancer is needed.

Optimal management of angiosarcoma has not yet been defined. The primary treatment remains wide resection of the tumor with negative margins. The retrospective national Finish study observed a greater number of local recurrences in patients when there were less than $1 \mathrm{~cm}$ tumor free margins and concluded that management of RAA of the breast currently involves a radical excision of the irradiated breast area. ${ }^{16}$ The role of adjuvant and neoadjuvant chemotherapy remains uncertain. Our case and case reported by Aljarrah et al. ${ }^{5}$ were the only two cases that used adjuvant chemotherapy after radical surgery. In three out of five reported cases surgery was the main and only treatment modality. Lately ECT joined as feasible therapy as there was reported $80 \%$ of objective response rate in 20 patients with advanced angiosarcoma that were treated with the use of ECT. ${ }^{17}$ In our case, ECT resulted in a complete response in the treated area but new tumors developed outside of the treated area in less than 2 months. Multimodal therapy seems promising in achieving longer survival as in our case, where the patient lived for almost 3 years after the initial diagnosis of RAA. In all other reported cases the follow up was up to one year or it was not defined, therefore we do not know if they had any recurrences after that time.

\section{Conclusions}

Women with breast cancer after skin-sparing mastectomy and immediate breast reconstruction rarely receive radiotherapy. Those who receive radiotherapy need regular long-term follow up and low threshold for biopsy of any suspicious lesions is mandatory. ECT proved as one of feasible modalities of treatment. 


\section{Acknowledgement}

This work was financially supported by Slovenian Research Agency (ARRS) [grant number P3-0003]. The investment was co-financed by the Republic of Slovenia and the European Regional Development Fund [Project SmartGene.Si].

\section{References}

1. Shah S, Rosa M. Radiation-associated angiosarcoma of the breast clinical and pathological features. Arch Pathol Lab Med 2016; 140: 477-81. doi: 10.5858/arpa.2014-0581-RS

2. Huang J, Mackillop WJ. Increased risk of soft tissue sarcoma after radiotherapy in women with breast carcinoma. Cancer 2001; 92: 172-80. doi: 10.1002/1097 142(20010701)92:1<172::aid-cncr1306>3.0.co;2-k

3. Cahan WG, Woodard HQ, Higinbotham NL, Stewart FW, Coley BL. Sarcoma arising in the irradiated bone: report of 11 cases. Cancer 1948; 1: 3-29. doi: 10.1002/1097-0142(194805)1:1<3::aid-cncr2820010103>3.0.co;2-7

4. Hanasono MM, Osborne MP, Dielubanza EJ, Peters SB, Gayle LB. Radiation-induced angiosarcoma after mastectomy and TRAM flap breast reconstruction. Ann Plast Surg 2005; 54: 211-4. doi: 10.1097/01. sap.0000134751.73260.3a

5. Aljarrah A, Nos C, Clough KB, Lefrere-Belda MA, Lecuru F. A case report on radiation-induced angiosarcoma of breast post skin-sparing mastectomy and reconstruction with transverse rectus abdominal muscle. Ecancermedicalscience 2014; 8: 402. doi: 10.3332/ecancer.2014.402

6. Yip C, Weiler-Mithoff E, Doughty JC, Lo SJ. Radiation-associated angiosarcoma after autologous breast reconstruction: report of two cases in a plastic surgery unit. Eur J Plast Surg 2019; 42: 513-16. doi: org/10.1007/s00238019-01536-8

7. Campana LG, Miklavcic D, Bertino G, Marconato R, Valpione S, Imarisio I et al. Electrochemotherapy of superficial tumors - current status: basic principles, operating procedures, shared indications, and emerging applications. Sem Oncol 2019; 46: 173-91. doi: 10.1053/j.seminoncol.2019.04.002.

8. Campana LG, Clover JP, Valpione S, Quaglino P, Gehl J, Kunte C, et al. Recommendations for improving the quality of reporting clinical electrochemotherapy studies based on qualitative systematic review. Radiol Oncol 2016; 50: 1-13. doi: 10.1515/raon-2016-0006.

9. Gehl J, Sersa G, Matthiessen LW, Muir T, Soden D, Occhini A, et al. Updated standard operating procedures for electrochemotherapy of cutaneous tumours and skin metastases. Acta Oncol 2018; 57: 874-82. doi: 10.1080/0284186X.2018.1454602

10. De Giorgi V, Santi R, Grazzini M, Papi F, Gori A, Rossari S et al. Synchronous angiosarcoma, melanoma and morphea of the breast skin 14 years after radiotherapy for mammary carcinoma. Acta Derm Venereol 2010; 90: 2836. doi: 10.2340/00015555-0841

11. Morgan EA, Kozono DE, Wang Q, Mery CM, Butrynski JE, Baldini EH, et al. Cutaneous radiation-associated angiosarcoma of the breast: poor prognosis in a rare secondary malignancy. Ann Surg Oncol 2012; 19: 3801-8. doi: 10.1245/s10434-012-2563-4

12. Fineberg S, Rosen PP. Cutaneous angiosarcoma and atypical vascular lesions of the skin and breast after radiation therapy for breast carcinoma. Am J Clin Pathol 1994; 102: 757-63. doi: 10.1093/ajcp/102.6.757

13. Biswas $T$, Tang $P$, Muhs $A$, Ling $M$. Angiosarcoma of the breast: a rare clinicopathological entity. Am J Clin Oncol 2009; 32: 582-6. doi: 10.1097/ COC.0b013e3181967f09

14. Bozikov K, Arnez T, Hertl K, Arnez ZM. Fat necrosis in free DIEAP flaps: incidence, risk, and predictor factors. Ann Plast Surg 2009; 63: 138-42. doi: 10.1097/sap.0b013e31818937d4

15. Palta M, Morris CG, Grobmyer SR, Copeland EM, 3rd, Mendenhall NP. Angiosarcoma after breast-conserving therapy: longterm outcomes with hyperfractionated radiotherapy. Cancer 2010; 116: 1872-8. doi: 10.1002/ cncr.24995
16. Salmien HS, Wiklund T, Sampo MM, Tarkkanen M, Pulliainen L, Böhling TO, et al. Treatment and prognosis of radiation-associted breast angiosarcoma in a nationwide population. Ann Surg Oncol 2020; 27: 1002-10. doi: 10.1245/s10434-019-08085-1

17. Campana LG, Kis E, Bottyán K, Orlando A, de Terlizzi F, Mitsala G, et al. Electrochemotherapy for advanced cutaneous angiosarcoma: A European register-based cohort study from the International Network for Sharing Practices of Electrochemotherapy (InspECT). Int J Surg 2019; 72: 34-42. doi: 10.1016/j.jjsu.2019.10.013 